\title{
Методологические аспекты формирования экологической политики в условиях городской среды
}

\author{
Л.Г. Селютина ${ }^{1}$, Е.В. Песоцкая ${ }^{2}$, А.Н. Черных ${ }^{2}$ \\ ${ }^{1)}$ Петербургский государственный университет путей сообщения Императора Александра I \\ Россия, 190031, Санкт-Петербург, Московский пр., 9 \\ ${ }^{2)}$ Санкт-Петербургский государственный экономический университет \\ Россия, 191023, Санкт-Петербург, ул. Садовая, 21 \\ E-mail: ya.slarisa@ya.ru, epes@mail.ru, andrey.chernykh9911@mail.ru
}

\begin{abstract}
Аннотация
В статье рассматриваются актуальные экологические составляющие современной градосистемы. Характеризуются особенности экологической политики, исследуются методологические основы ее формирования и реализации. Выделяются основные методологические принципы экологической политики, раскрывается их содержание и возможность использования в управленческом инструментарии экологической политики в условиях городской среды. Особое внимание уделяется исследованию проблемы развития сферы туризма в условиях экологизации экономики города. Раскрывается суть понятия «экологизация туристской деятельности», характеризуются направления ее реализации с выделением управленческого аспекта. Показана необходимость разработки планов и программ экологизации туризма на основе анализа взаимовлияния функционирования сферы туризма и состояния окружающей среды.
\end{abstract}

Ключевые слова: экологическая политика, методологические принципы, городское пространство, экологизация туристской деятельности.

Для цитирования: Селютина Л.Г., Песоцкая Е.В., Черных А.Н. 2020. Методологические аспекты формирования экологической политики в условиях городской среды. Экономика. Информатика. 47 (3): 473-482. DOI: 10.18413/2687-0932-2020-47-3-473-482.

\section{Methodological aspects of environmental policy formation in the urban environment}

\author{
L.G. Selyutina ${ }^{1}$, E.V. Pesotskaya ${ }^{2}$, A.N. Chernykh ${ }^{2}$ \\ ${ }^{1)}$ Emperor Alexander I St. Petersburg State Transport University, 9 Moskovsky pr., \\ St. Petersburg, 190031, Russia \\ ${ }^{2)}$ St. Petersburg State University of Economics, 21 Sadovaya St, 191023, Saint-Petersburg, Russia \\ E-mail: ya.slarisa@ya.ru, epes@mail.ru, andrey.chernykh9911@mail.ru
}

\begin{abstract}
The article discusses the current environmental components of a modern urban system. The features of environmental policy are characterized; the methodological foundations of its formation and implementation are investigated. The main methodological principles of environmental policy are highlighted; their content and the possibility of using environmental policy in management tools in the urban environment are revealed. Particular attention is paid to the study of the development of tourism in the context of greening the city's economy. The essence of the concept of "ecologization of tourist activity" is revealed, the directions of its implementation are described with the emphasis on the managerial aspect. The necessity of developing plans and programs for tourism greening based on the analysis of the mutual influence of the functioning of the tourism sector and the state of the environment is shown.
\end{abstract}

Keywords: environmental policy, methodological principles, urban space, ecologization of tourist activities.

For citation: Selyutina L.G., Pesotskaya E.V., Chernykh A.N. 2020. Methodological aspects of environmental policy formation in the urban environment. Economics. Information technologies. 47 (3): 473-482 (in Russian). DOI: 10.18413/2687-0932-2020-47-3-473-482. 


\section{Введение}

Первые два десятилетия XXI века ознаменовались необычным ростом городского населения, во много раз превышающим общий рост населения земного шара. При этом российская урбанизированная система характеризуется высокой долей городского населения 74,7 \% [Россия в цифрах, 2019]. Сегодня все большее значение для сохранения жизни людей и условий, необходимых для ее поддержания, приобретает комфортная городская среда, на что указывают многие зарубежные и отечественные исследователи [Bulgakova, 2018; Raven, 2020].

Вместе с тем экологические показатели состояния городских территорий оказывают существенное влияние на формирование и укрепление здоровья горожан, продолжительность их жизни и трудовой активности. Следовательно, здоровая и благоприятная для природы и человека городская среда обуславливает комфортность проживания людей, динамичное и сбалансированное социально-экономическое развитие города. Наряду с этим современный город с его социально-экономической и инженерно-технической инфраструктурой становится зоной проявления экологических проблем.

Устойчивое развитие городов при реализации градостроительной деятельности направлено на обеспечение градостроительными средствами благоприятных условий проживания населения, особенно на полноценную защиту окружающей среды от вредного влияния антропогенных факторов и разумное использование природно-ресурсного потенциала территории для целей удовлетворения потребностей ныне живущих и будущих поколений людей. [Титов, Ваганова, 2015; Maleeva, 2018]. Важно подчеркнуть, что поиск балансов при формировании городской среды в аспекте ее устойчивого и гармоничного развития осуществляется в системе градорегулирования с особым акцентом на экологические приоритеты, т. е. организацию среды с градоэкологической позиции.

Экология как современная наука в контексте государственного регулирования имеет реальное исполнение, фокусирующееся, прежде всего, в концепции и принципах регулирования природоохранной деятельности, что в частности проявляется в экологической политике. Однако следует отметить, что только лишь в последнее время во всех странах мира проявляется активный интерес к долгосрочным перспективам развития экологической политики со стороны органов государственного управления и местного самоуправления.

Актуальность экологической проблематики с учетом современных реалий, несомненно, нарастает. В контексте этого обстоятельства вполне обоснованно ориентироваться на экологическую политику, научно аргументированную и опирающуюся на современный методологический и методический инструментарий формирования и развития экосистемы. При этом также актуализируется задача по поиску управленческих инструментов, использование которых обеспечит эффективную реализацию экологической политики.

В связи с этим целью исследования, проведенного в данной статье, является определение сущности, особенностей, методологического базиса экологической политики в условиях городской среды и обоснование необходимости экологизации индустрии туризма как одного из определяющих факторов создания привлекательной и комфортной городской среды.

\section{Основные результаты исследования}

Исторически и содержательно термин «политика» основывается на древнегреческом понятии (др.-греч. тодıєки́ - город-государство), связанным с организацией совместной жизнедеятельности людей в обществе. Политика в традиционном понимании - искусство управления государством в целях обеспечения эффективного руководства и достижения общественного блага и стабильности, что тесно соотносится с главными трендами и принципами экополитики в современном мире.

Вопросы сущности экологической политики и ее роли в осуществлении управления природоохранной деятельности сегодня изучают многие российские и зарубежные исследователи [Катp I., 2003; Васильев А.Н., 2007]. Авторы настоящей статьи рассматривают экологическую политику как систему представлений о целях, принципах, приоритетах природоохранной направленности, базирующихся на документах, регламентирующих 
нормативный и правовой аспекты природосберегающей деятельности, и не входящих в противоречие с научно-техническими, социально-экономическими тенденциями мировой экономики, в рамках которых осуществляются взаимодействия человеческого общества и окружающей природной среды. Как иллюстрация приведенного высказывания ниже представлен рисунок.

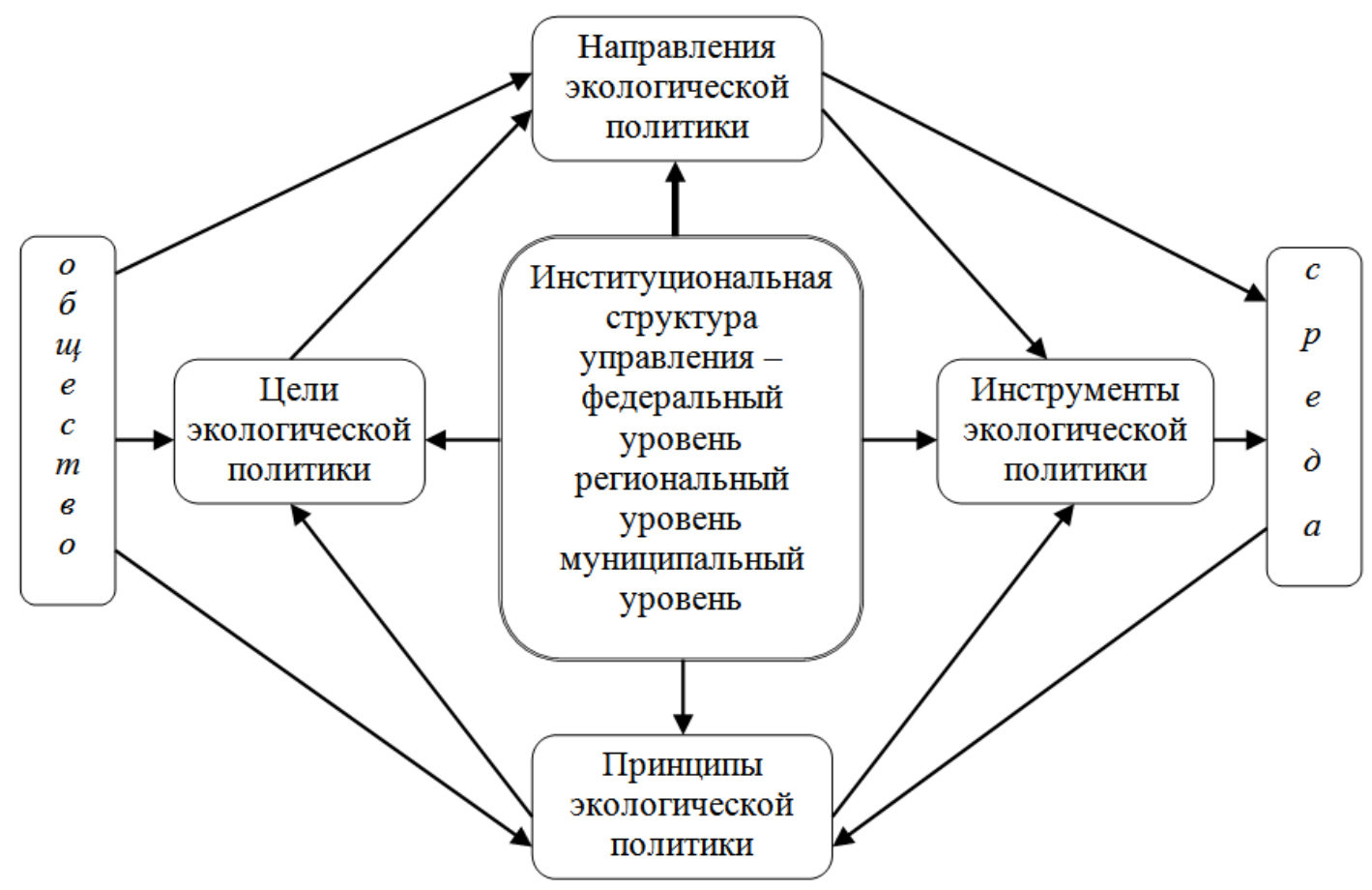

Рис. Теоретический аспект сущности экологической политики

Fig. Theoretical aspect of the essence of environmental policy

Следует особо подчеркнуть важность задачи большой сложности - выстраивание методологического и методического базиса экологической политики, позволяющей сформировать необходимые условия для обеспечения устойчивого развития экономики, усиления ее потенциала и повышения удовлетворенности населения качеством жизни.

Как считают некоторые эксперты и ученые, работающие в области экологической экономики, решение этой значимой задачи возможно с помощью важнейшего требования современности - экологизации бизнес-процессов [Бессонов, 2016; Egorova, 2017], предполагающей планомерное внедрение и конкретизацию экологических императивов в правовых нормах ведения бизнеса.

Анализ отечественных и зарубежных авторских подходов к понятию «экологизация» показывает, что значительная часть определений к его трактовке ориентируется на всю экономическую систему и охватывает действия в направлении активизации «зеленой» трансформации экономики, формирующей актуальную основу и эволюционную перспективу ее устойчивого развития. Однако, как представляется авторам статьи, целесообразно рассматривать экологический подход относительно конкретных элементов экономической сферы общественной жизни. В частности, туристская деятельность заслуживает особого внимания с позиции экологической безопасности городской среды.

Методологическую базу экологической политики формируют принципы, позволяющие учесть фундаментальные аспекты экологизации, а также специфику применения научных подходов в исследовании этого процесса. Анализ соответствующей современной литературы по проблеме исследования [Каранда, 2018; Frolova, 2019] позволяет сформулировать методологические принципы, при интегрированном использовании которых возможно формирование целостной и логически выверенной экологической политики в условиях городской среды. 
Ключевым методологическим принципом является принцип системности, неразрывный с методологической предпосылкой формирования экологической политики системным подходом, применение которого дает возможность рассмотрения объекта изучения как сложной, целостной системы с множеством взаимосвязанных и качественно разнообразных элементов. При этом системный подход в сочетании с интегративным видением экологической деятельности и с учетом административно-территориальных интересов может быть реализован по следующим направлениям, ориентированным на выявление, исследование и решение проблем экологии:

- в контексте необходимости перехода к устойчивому природосовместимому развитию современной градосистемы экополитика проявляется как совокупность намерений и принципов относительно экосистемы, выступающей в качестве системного объекта;

- исследование экологических составляющих градосистемы во взаимовлиянии и взаимосвязанности с социальными и экономическими компонентами, агрегированными в сложную социо-эколого-экономическую систему;

- учитывая важность преодоления современных экологических проблем, экополитика базируется на комплексности взаимосвязанных мероприятий, направленных на обеспечение экологических целей и задач, причем целесообразность и практическая значимость этих действий обусловлена непосредственно их системностью.

Вместе с тем экологическая политика характеризуется как исходный компонент иерархической организации экологического менеджмента, содержащей последовательно структурированные и системно связанные элементы (начиная с разработки политики и заканчивая оперативными решениями по ее реализации), которые в совокупности генерируют многоуровневые иерархии, адекватные переходу от наиболее общего к конкретному (частному).

Другим принципом, неразрывно связанным с принципом системности, является принцип целеполагания. Вопрос формулировки целей и задач экологической политики является объектом исследования в трудах многих зарубежных и отечественных ученых [Каранда, 2018; Raven, 2020]. Анализ изложенных в них точек зрения исследователей позволил вычленить три группы целевых установок в области экологической политики:

- рациональное использование природного разнообразия и сохранение естественных экосистем;

- достижение гармонии между экологическим состоянием окружающей среды и реализацией результатов научно-технического и социально-экономического развития урбанизированных территорий;

- комплексная организация процесса целенаправленной экологизации секторов экономики и видов экономической деятельности, имеющая целью их выход на траекторию развития в условиях зеленой экономики.

Представленные группы следует рассматривать с позиции дальнейшей дифференциации целевых установок. При этом выполняется процедура, предопределенная методологической концепцией системного подхода - формирование внутри каждой группы блока подцелей, которые в последовательном порядке переходят в конкретные практические задачи.

Большое значение в формировании современной экологической политики имеет принцип гармонизации интересов. Реализация этого принципа направлена на принятие своевременных, правильных управленческих решений в области природопользования и экологии, не создающих экологических угроз и не вступающих в противостояние с интересами общества. Иногда принцип гармонизации в терминологическом контексте отождествляют с принципом баланса интересов. Помимо очевидного сходства этих терминов следует отметить и существующие отличия, связанные с конкретными задачами исследования. Обычно баланс интересов рассматривают не обособленно, а в единой связи с поставленными целями, реализация которых возможна на основе построения некоторой балансовой модели. С экологической позиции применение данного моделирования не представляется возможным ввиду многоаспектности экологической политики, а также наличия неопределенности в ходе 
реализации экологических процессов и экологических последствий. В связи с этим в данном случае совершенно правомерно стремиться к согласованности целей и опираться именно на гармонизацию интересов, осуществляющуюся без четкого формализованного описания, в свою очередь, присущую модели балансовой технологии.

Не менее важным принципом современной экологической политики является принции инновационности как основополагающий вектор, задающий направление развития исследуемой сферы. Его актуальный фон очень высок и обусловлен, прежде всего, разработанной и в настоящее время реализуемой государственной концепцией проектирования инновационных преобразований во всех сферах общественной жизни, в т. ч. в сфере экологии и охраны окружающей среды. Действие принципа инновационности распространяется в рамках процесса экологизации на все отрасли экономики хозяйственного комплекса, проявляется в использовании наилучших доступных технологий, снижающих техногенную нагрузку на окружающую среду и обуславливающих внедрение системы зеленой экономики.

В качестве другого принципа экологической политики следует выделить принцип результативности, внедрение которого означает, что при формировании и реализации экологической политики ее разработчики должны исходить из необходимости достижения заданных результатов, касающихся как экологической, так и социально-экономической ситуации в целом. При этом важным является наличие у результатов четко обозначенного контента, который может иметь количественную и качественную характеристику. При правильной оценке количественные параметры благодаря достаточной точности служат аналитическим базисом формирования достоверных динамических рядов, необходимых для оценки прогноза и реализации рациональных управленческих решений. В соответствии с этим следует максимизировать число показателей результатов, имеющих количественные характеристики.

Напротив, качественные параметры нужно минимизировать, т. к. они не поддаются строгому количественному измерению, обладают низкой точностью и их оценивают по итогам наблюдения за результатами, т. е. субъективно, на основе экспертных оценок. Поэтому у многих исследователей возникают сомнения в верности инструментальной базы (алгоритма и процедур оценки) метода экспертного оценивания.

Немаловажно отметить и тот факт, что качественные показатели являются опережающими индикаторами, которые, влияя на конечные результаты, как бы предупреждают о вероятных отклонениях количественных параметров, и если измерять качественные показатели, то с большей вероятностью можно достичь и требуемый количественный результат. Поэтому при формировании и оценке результатов экологической политики использование качественных показателей также неизбежно, однако, учитывая универсальность экспертного метода, следует сфокусироваться на совершенствовании его алгоритма и процедур поддержки принятия решения или выбора.

Необходимо обратить особое внимание на соблюдение принципа организационного единства. На современном этапе важную роль в реализации эколого-хозяйственных приоритетов играет гибкая, рационально сбалансированная организационная среда, базирующаяся на единых подходах и нормах, формирующаяся с учетом четкого распределения ответственности за несоблюдение экологических нормативов и требований. С учетом этого, экологическая политика должна ориентироваться на организационные условия, обеспечивающие менеджмент исполнения природоохранных мероприятий, целенаправленную практическую реализацию различного уровня программ экологической направленности, соответствующих планов оперативного, тактического и стратегического уровней. Организационная основа экологической политики в виде вышеперечисленных ее элементов соединяется с процессом выстраивания специальной регуляторной среды и инструментов с функцией поддержания заданных элементов в статусе однозначности, логичности и достаточности.

Следующий принцип, базирующийся на структурировании накопленных экологических знаний, их систематизации и обобщения, можно сформулировать как принцип 
открытого обсуждения, устанавливающий следующее положение: обсуждение актуальных вопросов охраны окружающей среды, природопользования и экологической безопасности должно осуществляться с активным участием общественных организаций и граждан. Применение данного принципа предполагает использование сети Интернет и различных приложений мобильных социальных сетей. Существенный результат может дать организация и проведение публичных слушаний, общественных обсуждений природоохранных мероприятий, планов, программ и проектов.

К числу наиболее быстроразвивающихся рыночных сфер в городской среде относится современный туризм, развитие которого характеризуется возникновением проблем, связанных с процессом экологизации туризма, необходимостью актуализации в туристском секторе парадигмы экологизации социально-экономического пространства, что, безусловно, предусматривает применение адаптивного инструментария управления.

Важно подчеркнуть, что расширение сферы взаимодействия природы и человека объективно ведет к усилению роли экологически устойчивых системных процессов в обществе. В современной науке к таким процессам относится экологизация туристской деятельности, которая как научная категория в последние годы получила широкое распространение. Однако следует отметить, что использование данной категории не всегда сопровождается раскрытием и уточнением ее смысловой нагрузки, при этом существующие подходы к ее трактовке, в целом не имея принципиальных отличий, осуществляются с разных позиций [Черных, 2017; Kamp, 2003]: рассматривают ее обобщенно или фрагментарно по отдельным составляющим процесса экологизации.

Учитывая результаты анализа точек зрения представителей зарубежной и отечественной научной школы по исследуемому вопросу под экологизацией туристской деятельности, на наш взгляд, следует понимать длительный инновационно-технологический процесс постепенного внедрения новых туристских практик с экологически чистыми и безопасными составляющими, способствующих устойчивому развитию туризма с минимальным негативным влиянием на экологию.

В этой связи к основным задачам экологической политики в сфере туризма, поддерживающей и способствующей повышению результативности экологизации экономики на национальном, региональном и местном уровнях можно отнести:

- мотивация граждан к здоровому образу жизни, что необходимо для развития личности и общества в целом;

- обеспечение сохранения целостности природных ресурсов и наращивания природно-ресурсного потенциала российских территориальных образований;

- активизация развития туристской индустрии, выступающей в качестве одного из наиболее динамично развивающихся и эффективных секторов экономической системы и являющейся катализатором экономического и социального развития территорий;

- предоставление потребителям в сфере туристских услуг туристской продукции с экологически чистыми составляющими, что имманентно присуще экотуризму;

- развитие важнейшей составляющей современной сферы туристской индустрии системы охраны окружающей природной среды;

- создание условий для формирования эффективных управленческих решений в области проектирования и организации туристско-рекреационных территорий при помощи активного участия реальных пользователей территории - местных жителей и городских сообществ, и другие задачи.

Для решения данной группы задач требуется разработка и применение соответствующих правил и процедур выработки, принятия и корректировки оперативных, тактических и стратегических управленческих решений. Причем каждый из этих механизмов управления должен строиться с учетом сложности, многофакторности экологических проблем и ситуаций, и неприемлемости их решения посредством лишь общезначимых положений теории менеджмента. В этой связи представляется целесообразным при формировании экологической политики ориентировать универсальный управленческий инструментарий в 
сторону применения экологического подхода с акцентом на его базовых принципах, предметно исследовав их с точки зрения поиска эффективных управленческих решений, учитывающих снижение экологического риска и экологического дефицита территории.

Анализируя сложившуюся практику решения тех или иных проблем экологизации экономики, важно отметить следующие направления современного процесса экологизации городской туристской деятельности:

- разработка и реализация стратегии и приоритетных направлений развития процесса экологизации туризма как составной части концепции экологически сбалансированного, устойчивого развития экономики;

- развитие инновационных процессов в туристской индустрии, на основе применения ресурсосберегающих технологий и инновационно-технических решений, сопровождаемое функционированием взаимосвязанных обслуживающих комплексов;

- формирование экологически ориентированной городской транспортной инфраструктуры, прежде всего за счет использования в туристском обслуживании экологически чистых («зеленых») транспортных средств;

- организация экологически безопасной системы обращения с бытовым и промышленным мусором, образующимся при функционировании турорганизаций;

- развитие системы менеджмента экологического качества экономики в контексте внедрения методологии (принципов и методов) управления качеством экологизации туристской деятельности;

- создание интеграционной системы организационного развития процесса экологизации хозяйственной сферы экономики градосистемы и индустрии туризма с широким охватом всех ее элементов: разработкой необходимых организационных схем, правил, норм и стандартов; идентификацией субъектных позиций в структурной схеме; организацией системы обучения экологической безопасности (экологии) с подготовкой организационноуправленческих кадров в сфере экологического туризма.

Реализация программ по экологической ориентации туризма и планов экологизации туристской деятельности обеспечивается действием множества факторов и условий, среди которых особое место занимает объективно существующая взаимообусловленность процесса развития индустрии туризма и состояния природной среды в любом территориальном структурном компоненте.

Для получения адекватной оценки степени и характера такого взаимодействия целесообразно предложить следующий алгоритм, состоящий из последовательных этапов.

На начальном этапе построения алгоритма составляется перечень индикаторов для оценки уровня развития процесса экологизации. Система таких данных в большинстве случаев включает количественные характеристики существующей ситуации:

- количество организаций туристского профиля, работающих в рамках нормативноправовых условий экологического туризма;

- число потребителей услуг эколого-туристских организаций;

- величина финансовых средств, направляемых на экологизацию туристского бизнеса;

- размер расходов по внедрению инновационных ресурсосберегающих экологических технологий в туризме и другие показатели.

В дополнение к ним могут быть использованы качественные параметры, вычисляемые с помощью метода экспертных оценок.

Затем переходят к этапу сбора показателей, их обработки, фиксирования и приведения в систему с последующим изучением изменений анализируемых показателей во времени.

Построение шкалы оценки динамических изменений по каждому анализируемому показателю выполняется уже на следующем этапе. Важной характеристикой подобной шкалы является точность, которая обуславливается действиями, проявляемыми ее разработчиками в направлении логического следования и рационального мышления. Среди аналитических методов, применяемых в таких исследованиях, основными и наиболее распространенными являются: метод факторного анализа, метод анализа иерархий, метод экстраполяции, метод экспертной оценки, метод логического моделирования, причем получило широкое применение также изучение прошлого опыта. 
Далее следует этап непосредственного измерения скорости изменения (динамики) оцениваемых показателей. Помимо этой важной процедуры, на данном этапе также выполняется анализ качественных показателей со вниманием к изменению уровней динамических рядов.

Принципиально важен следующий этап, в рамках которого строится система баллов косвенных оценок, используемых с целью описания конкретных изменений относительно каждого из анализируемых параметров. Для выполнения этого процесса, как и других оценочных процедур, применяемых в представленном алгоритме, может быть использован метод экспертных оценок с учетом определения параметров весомости (значимости) показателей, интегрирующих в себе характеристики пространства взаимодействия индустрии туризма и окружающей природной среды.

В целях предотвращения экспертных ошибок и придания результатам экспертного оценивания несомненной достоверности и обоснованности важно выполнять следующие требования к экспертам и организации процесса проведения экспертной оценки:

- для надежного обоснования принятия решения важно привлекать к участию в экспертных оценках достаточный численный состав экспертной группы, не допускать принятия решений, основанного на использовании одного или двух мнений, независимо от их организационно-управленческого ранга;

- специалисты, привлекаемые в роли экспертов, должны обладать профессиональной компетентностью, независимостью суждений и заинтересованностью в объективных результатах экспертизы;

- реализация возможности ознакомления с прошлым опытом экспертов с целью его подробного анализа и предотвращения на его основе возможных экспертных ошибок, а также согласования точек зрения и выработки единой позиции между экспертами.

Заключительный этап базируется на работе с результатами оценок, выставленных экспертами, тем самым отражается интегральный уровень расчетов. Причем объективно возрастает сложность таких расчетов, предполагающих учет степени значимости оцениваемых параметров, их возможностей адекватно выразить интенсивность взаимообусловленности экологических факторов и состояния городской среды и функционирования туристской индустрии. Нередко для решения подобных задач используется метод весовых коэффициентов, точность определения которых зависит от компетентности и опыта экспертов, и их умения методически сформировать систему взаимовлияния разнообразных качественных факторов.

Как видно из вышеприведенного алгоритма, при реализации его составных компонентов уместно исходить из возможности применения метода экспертного оценивания, что вполне правомерно. В поддержку этого тезиса следует отметить, что степень взаимодействия (взаимосвязи) определенных явлений действительности имеет качественную характеристику и не поддается измерению иным способом, а именно только с помощью экспертных оценок. Фактически экспертная оценка является попыткой количественно измерить показатель, отражающий системную глубину качественных показателей, а переход качества в количество относительно практики принятия управленческих решений или выполнения экономических обоснований, а также методик, их регламентирующих, в большинстве случаев подкрепляется заключениями экспертов.

\section{Заключение}

Вышеизложенное позволяет сформулировать следующие выводы.

Во-первых, в работе исследованы методологические принципы формирования экологической политики городского развития, средством реализации которых является набор определенных инструментов, регулирующих экологическое равновесие между окружающей природной средой и человеком (обществом). Принято различать две группы рычагов воздействия на состояние экологии в стране (регионе): прямые и косвенные. Первая группа включает инструменты прямого регулирования, предполагающие меры административноправового воздействия, вторая же - основана на организационно-экономических методах 
регулирования. Обе инструментальные группы регулирования многочисленны и достаточно многообразны, однако, к сожалению, преждевременно говорить о результативном инструментальном обеспечении экологической политики. Хотя достаточно хорошо и глубоко проработаны теория и методология отдельных компонентов экологического менеджмента, до сих пор на стадии обсуждения находятся вопросы выявления и оценки степени проявления стимулирующего фактора во многих инструментах воздействия, в т. ч. не вполне ясна их взаимосвязь, сочетаемость и комплементарность. Вместе с тем возникают вопросы об актуальном значении многих инструментов и их практическом использовании при решении конкретных экологических задач. Фактически сегодня также не до конца ясны итоговые последствия в отношении многих принятых и реализованных в последнее время экологически значимых управленческих решений. При этом современные проблемы экологии не систематизированы, а их идентификация и решения часто имеют фрагментарное представление. Касаясь этих аспектов исследуемой проблемы, важно отметить, что только всесторонние и полные исследования, базирующиеся на объективном теоретикометодологическом обосновании, включающем в себя наиболее значимые элементы системного подхода, могут обеспечить адекватное решение данных вопросов.

Во-вторых, важным элементом представления об экологически устойчивом развитии экономики города является экологизация туризма, которая с позиции современного менеджмента несет в себе много вопросов, требующих безотлагательного решения. Одним из таких открытых вопросов выступает систематический анализ и оценка взаимообусловленности, взаимосвязи окружающей природной среды (экологических факторов) и сферы туризма (перспектив его развития) с отражением их непосредственных результатов в принимаемых управленческих решениях. Именно его проработке и решению должно уделяться значительное внимание, т. к. благодаря этому формируются основные подходы и механизмы успешной реализации экологической политики, основы сбалансированного развития которой интегрируются и в индустрию туризма, остающегося одним из наиболее динамично развивающихся и эффективных секторов экономики современной градосистемы.

\section{Список литературы}

1. Бессонов М.С., 2016. Социальное жилье - стратегическое направление развития строительства в регионах России. Международный журнал прикладных и фундаментальных исследований, 6-1: 142-144.

2. Васильев А.Н. 2007. Совершенствование организации управления инвестиционными процессами инновационной деятельности предприятий строительного комплекса. Управление инвестициями и инновациями, 1 (1): 46-55.

3. Каранда А.В. 2018.Особенности реализации экологической политики в условиях городской среды. Аллея науки, 6 (22): 332-335.

4. Россия в цифрах - 2019, 2019. М., Росстат-М., 549.

5. Титов А.Б., Ваганова О.В., 2015. Методический подход к определению перспективных отраслей региона в условиях турбулентности экономики. Научный результат. Серия: Экономические исследования, 1-3: 56-63. DOI: 10.18413/2409-1634-2015-1-3-56-63.

6. Черных А.Н., 2017. Развитие сферы туризма в условиях экологизации экономики: Дисс. на соиск. уч. степ. канд. экон. наук. СПб.: СПбГЭУ.

7. Bulgakova K.O., 2018. Basics of investment projects selection for the implementation of regional investment programs in the sphere of social house building. Espacios, 39 (26).

8. Egorova M., 2017. Modeling of investment processes in the sphere of social house building. Innovations in science and education. International Conference Proceedings. Central Bohemia University, pp. 67-72.

9. Frolova N.N., 2019. Acceleration of regional housing development in Russia on the basis of industrial housing construction modernization. E3S Web of Conferences, 97: 06003. DOI: $10.1051 / \mathrm{e} 3$ sconf/20199706003.

10.Kamp I., 2003. Urban environmental quality and human well-being: Towards a conceptual framework and demarcation of concepts; a literature study. Landscape and Urban Planning, 65 (1-2): 5-18 (in Russian).

11.Maleeva T., 2018. Analysis and evaluation of financial resources of social housing construction in city. Materials Science Forum, 931: 1118-1121. 
12.Raven R., 2020. Nurturing nature: Exploring socio-spatial conditions for urban experimentation. Environmental Innovation and Societal Transitions, 34: 7-25.

\section{References}

1. Bessonov M.S., 2016. Social housing - a strategic direction for the development of construction in the regions of Russia. International Journal of Applied and Basic Research, 6-1: 142-144 (in Russian).

2. Vasiliev A.N., 2007. Improving the organization of management of investment processes of innovation activities of enterprises of the construction complex. Investment and Innovation Management, 1 (1): 46-55 (in Russian).

3. Karanda A.V., 2018. Features of the implementation of environmental policy in an urban environment. Alley of Science, 4-6 (22): 332-335 (in Russian).

4. Russia in numbers - 2019 [Moscow]. 2019: Moscow, Rosstat-M., 549.

5. Titov A.B., Vaganova O.V., 2015. A methodological approach to identifying promising industries in a region in a turbulent economy. The scientific result. Series: Economics Research, 1-3: 56-63. DOI: 10.18413/2409-1634-2015-1-3-56-63.

6. Chernykh A.N., 2017. Development of tourism in the context of greening the economy: Candidate's thesis, St.-Petersburg: Publishing House of SPbGIEU (in Russian).

7. Bulgakova K.O., 2018. Basics of investment projects selection for the implementation of regional investment programs in the sphere of social house building. Espacios, 39 (26).

8. Egorova M., 2017. Modeling of investment processes in the sphere of social house building. Innovations in science and education. International Conference Proceedings. Central Bohemia University, pp. $67-72$.

9. Frolova N.N., 2019. Acceleration of regional housing development in Russia on the basis of industrial housing construction modernization. E3S Web of Conferences, 97: 06003. DOI: $10.1051 / \mathrm{e} 3$ sconf/20199706003.

10.Kamp I., 2003. Urban environmental quality and human well-being: Towards a conceptual framework and demarcation of concepts; a literature study. Landscape and Urban Planning, 65 (1-2): 5-18 (in Russian).

11.Maleeva T., 2018. Analysis and evaluation of financial resources of social housing construction in city. Materials Science Forum, 931: 1118-1121.

12.Raven R., 2020. Nurturing nature: Exploring socio-spatial conditions for urban experimentation. Environmental Innovation and Societal Transitions, 34: 7-25.

\section{ИНФОРМАЦИЯ ОБ АВТОРАХ}

Селютина Лариса Григорьевна, доктор
экономических наук, профессор, профессор
кафедры экономики и менеджмента в
строительстве Петербургского государственного
университета путей сообщения Императора
Александра I, Санкт-Петербург, Россия

Песоцкая Елена Владимировна, доктор экономических наук, профессор, профессор кафедры экономики и менеджмента в сфере услуг Санкт-Петербургского государственного экономического университета, Санкт-Петербург, Россия

\section{INFORMATION ABOUT THE AUTHORS}

Larisa G. Selyutina, Doctor of Economics, Professor, Professor of the Department of Economics and Management in Construction of Emperor Alexander I St. Petersburg State Transport University, Saint Petersburg, Russia

Elena V. Pesotskaya, Doctor of Economics, Professor, Professor of the Department of Economics and Management in the Service Sector, St. Petersburg State University of Economics, Saint Petersburg, Russia

Andrey N. Chernykh, Candidate of Economic Sciences, Associate Professor, Associate Professor of the Department of Economics and Management in the Service Sector, St. Petersburg State University of Economics, Saint Petersburg, Russia 